\title{
Propagation in a thermal graviton background
}

\author{
Daniel Arteaga* \\ Departament de Física Fonamental, Universitat de Barcelona, Av. Diagonal 647, 08028 Barcelona, Spain \\ Renaud Parentani ${ }^{\dagger}$ \\ Laboratoire de Physique Théorique, CNRS UMR 8627, Université Paris XI, 91405 Orsay Cedex, France
}

\author{
Enric Verdaguer \\ Departament de Física Fonamental and CER en Astrofísica, Física de Partícules i Cosmologia, Universitat de Barcelona, \\ Av. Diagonal 647, 08028 Barcelona, Spain \\ (Received 20 November 2003; published 18 August 2004)
}

\begin{abstract}
It is well known that radiative corrections evaluated in nontrivial backgrounds lead to effective dispersion relations which are not Lorentz invariant. Since gravitational interactions increase with energy, gravity-induced radiative corrections could be relevant for the trans-Planckian problem. As a first step to explore this possibility, we compute the one-loop radiative corrections to the self-energy of a scalar particle propagating in a thermal bath of gravitons in Minkowski spacetime. We obtain terms which originate from the thermal bath and which indeed break the Lorentz invariance that possessed the propagator in the vacuum. Rather unexpectedly, however, the terms which break Lorentz invariance vanish in the high three-momentum limit. We also found that the imaginary part, which gives the rate of approach to thermal equilibrium, vanishes at one loop.
\end{abstract}

DOI: $10.1103 /$ PhysRevD.70.044019

PACS number(s): 04.60.- m, 04.62.+v, 11.10.Wx

\section{INTRODUCTION}

In recent years modified dispersion relations which break Lorentz invariance have appeared in different contexts of gravitational physics.

On the one hand, they appeared in several works which address the so-called trans-Planckian problem [1-3]. In black hole physics the modes responsible for Hawking radiation reach arbitrarily high energies near the black hole horizon when measured by a free-falling observer. This observation has lead some authors to study the robustness of Hawking radiation when modifying (from the outset) the dispersion relation beyond the Planck scale [4-7]. A similar problem appears in inflationary cosmology: the modes responsible for the large-scale structure had length scales much smaller than the Planck length in the early stages of inflation. Therefore it is of interest to determine to what extent the properties of the fluctuation spectrum are sensitive to modifications of the dispersion relation at the Planck scale [8-11]. In these works, the introduction of a nontrivial dispersion relation has been originally suggested by formal analogies with condensed matter [4,12-15]. However, the main physical motivation is that unknown effects of quantum gravity (such as radiative corrections) might introduce nontrivial dispersion relations in these contexts. Following 't Hooft's [16] observation that strong gravitational interactions in the nearhorizon region might alter the semiclassical description of black hole evaporation, a first dynamical realization of this line of thought has been pursued in Refs. [17,18].

On the other hand, there have been hints from high-

\footnotetext{
*Electronic address: darteaga@ub.edu

${ }^{\dagger}$ Electronic address: Renaud.Parentani@th.u-psud.fr

‡Electronic address: verdague@ffn.ub.es
}

energy astroparticle physics that we could already see the effects of a violation of the Lorentz symmetry. In particular modified dispersion relations have been used in order to provide an explanation for observations of ultrahigh-energetic cosmic rays (with energies higher than $10^{20} \mathrm{eV}$ ) beyond the Greisen-Zatsepin-Kuzmin (GZK) cutoff [19-22]. As in the trans-Planckian problem, several dispersion relations have been exploited, inspired by various approaches to quantum gravity $[23,24]$, string theory [25], noncommutative field theory [26], variation of couplings [27], and multiverses [28]. Modified dispersion relations often break the energy degeneracy for a given three-momentum: the particle energy can become helicity dependent $[23,24]$ and light can become birefringent $[29,30]$. A popular form for these modified dispersion relations below the Planck scale is, in the cosmological rest frame,

$$
E^{2}=m^{2}+|\mathbf{p}|^{2}+\sum_{n \geqslant 3} \eta_{n} \frac{|\mathbf{p}|^{n}}{M_{\mathrm{Pl}}^{n-2}},
$$

where $M_{\mathrm{Pl}}$ is Planck's mass and $\eta_{n}$ are coefficients of order 1 if the Planck mass is the relevant scale. Jacobson et al. have studied the constraints on the possible values of the parameters $\eta_{n}$ based on current astrophysical observational data [31-34]. It is important to notice that both the real and imaginary parts $\eta_{3}$ are already observationally constrained to be much smaller than 1 . This shows that the "natural" assumption that the $\eta_{n}$ should be of order 1 is perhaps too naive. The possibility of quadratic modifications of the dispersion relation is also strongly constrained [29,30].

As we learned from particle physics, Lorentz invariance is a key element in renormalized quantum field theory $[35,36]$. Nevertheless, it might be broken by quantum gravity at a fundamental level, or, alternatively, it may be broken in an effective way in nontrivial backgrounds only. Indeed in 
backgrounds which possess a preferred reference frame, radiative corrections to self-energies might contain terms which effectively break the (local) Lorentz invariance-i.e., the Lorentz invariance in the tangent plane. Let us emphasize that this second possibility does not imply any kind of fundamental breaking of the Lorentz symmetry or any new physics. Rather by "effective breaking of the Lorentz invariance" we mean that radiative corrections to the dispersion relation may contain terms which depend on vector or tensor fields characterizing the background.

In this paper we explore a model in the framework of this second alternative. We consider the propagation of a scalar particle immersed in a thermal bath of gravitons. Our aim is to determine how the inertia of the thermal bath affects the propagation of the scalar particle through radiative corrections. To this end, we shall compute the thermal corrections to its self-energy. The real part of the self-energy gives the thermal mass shift and might introduce as well a nontrivial dispersion relation [37]. The imaginary part instead gives the rate of approach to thermal equilibrium [38].

In fact it is well known that quantum effects evaluated in nontrivial backgrounds induce non-Lorentz-invariant terms in the dispersion relation. For instance, the self-energy of a charged fermion immersed in a thermal bath of photons has been computed in Refs. [37,39-41], and the effects of the thermal bath on the speed of light have been studied in Refs. [42-44]. Let us mention also that electromagnetic interaction in curved spacetimes also leads to modifications of the dispersion relations. The effect of QED vacuum polarization on the speed of light in nontrivial spacetimes has been studied in Refs. [45-49]. However, most calculations done so far do not concern gravitational interactions. Gravity-induced corrections should be dominant at energies approaching the Planck scale, hence relevant for the situations concerned with the trans-Planckian problem. Additionally notice that gravitational interactions are universal and not limited to charged particles.

Two important points concerning our approach should be mentioned. On the one hand, since gravitational interactions are nonrenormalizable, the system of the scalar field coupled to gravity should be conceived as an effective field theory $[36,50]$. Although the full theory is nonrenormalizable, lowenergy predictions which do not depend on the Planck scale behavior of gravity can be extracted [51-53]. On the other hand, since thermal corrections do not affect the ultraviolet properties of the theory, thermal field theory fits well with the effective theory approach. At a technical level, we shall incorporate the thermal effects through the real-time description of thermal field theory [54-56], which can be seen as a particular application of the closed-time path (CTP) method in field theory [57-59].

We should also emphasize that we shall compute only the lowest-order corrections. Hence, we deal with free gravitons; i.e., we neglect the backreaction of the scalar field on the metric perturbations. The modifications to the metric propagator can be computed both in the framework of stochastic gravity [60-69] or in the large- $N$ limit of quantum gravity [70] (both approaches can be seen to be equivalent [71,72]). A computation along these lines (but in the semiclassical approximation) has been carried out by Borgman and Ford [73]. They study the fluctuations in the focusing of a bundle of geodesics, propagating in a spacetime with metric perturbations induced by a thermal scalar field. To our knowledge, the higher-order corrections to the self-energy have not yet been computed in the quantum field theoretical framework.

Gravity-mediated modifications of the dispersion relation have been also considered by Burgess et al. [74]. Working in the context of brane-world scenarios, these authors compute the change to the dispersion relation of photons and fermions generated by interaction with four-dimensional effective gravitons. In that case the primary source of violation of the Lorentz symmetry is the modification of the dispersion relation of effective four-dimensional gravitons induced by certain extra-dimensional configurations. Similarly to our case, this modification is communicated to brane-bound particles through gravitational interactions.

The plan of the paper is the following: In Sec. II we introduce the action for the system considered-namely, a scalar field coupled to gravity. In Sec. III we compute perturbatively the leading correction to the self-energy of the scalar field at zero temperature. In Sec. IV we assume that the scalar field and gravitons are in thermal equilibrium at a given temperature and compute the corrections to the retarded self-energy. Finally, in Sec. V we analyze the results and discuss the physical significance of the terms which break Lorenz invariance. The Appendixes contain reference material as well as some technical details of the calculation. In Appendix A we present the Feynman rules for the scalar field coupled to linearized gravity. In Appendix B we give the results of the calculation of some integrals in dimensional regularization. In Appendix $\mathrm{C}$ we give a brief account of the real-time approach to thermal field theory. Appendix D is mostly technical and is devoted to the calculation of certain integrals that appear in the calculation of the thermal contribution to the self-energy.

Throughout this paper we shall use a system of units with $\hbar=c=k_{\mathrm{B}}=1$. The signature of the metric will be $(-,+,+,+)$.

\section{SYSTEM}

We consider a minimally coupled real scalar field $\phi$ of mass $m$ propagating in a spacetime characterized by a metric $g_{\mu \nu}$. The action for the field is

$$
S_{\phi, g}=-\int \mathrm{d}^{4} x \sqrt{-g}\left(\frac{1}{2} g^{\mu \nu} \partial_{\mu} \phi \partial_{\nu} \phi+\frac{1}{2} m^{2} \phi^{2}\right),
$$

and the action for the metric is

$$
S_{g}=\frac{2}{\kappa^{2}} \int \mathrm{d}^{4} x \sqrt{-g} R,
$$

where $R$ is the Ricci scalar, $g$ is the determinant of the metric, and $\kappa=\sqrt{32 \pi G}=\sqrt{32 \pi} L_{\mathrm{Pl}}$ is the gravitational coupling constant, with $G$ being Newton's constant and $L_{\mathrm{Pl}}$ being Planck's length. Assuming that the metric is a small perturbation of Minkowski spacetime, $g_{\mu \nu}=\eta_{\mu \nu}+\kappa h_{\mu \nu}$, the com- 
plete action $S=S_{\phi, g}+S_{g}$ can be decomposed into the free scalar field, graviton, and interaction actions as $S=S_{\phi}+S_{h}$ $+S_{\text {int }}$. These actions, expanded in powers of $\kappa$, are

$$
\begin{aligned}
S_{\phi}= & \int \mathrm{d}^{4} x\left(-\frac{1}{2} \partial_{\mu} \phi \partial^{\mu} \phi-\frac{1}{2} m^{2} \phi^{2}\right), \\
S_{h}= & \int \mathrm{d}^{4} x\left(-\frac{1}{2} \partial^{\alpha} h^{\mu \nu} \partial_{\alpha} h_{\mu \nu}+\partial_{\nu} h^{\mu \nu} \partial^{\alpha} h_{\mu \alpha}\right. \\
& \left.-\partial_{\mu} h \partial_{\nu} h^{\mu \nu}+\frac{1}{2} \partial^{\mu} h \partial_{\mu} h\right)+O(\kappa), \\
S_{\mathrm{int}}= & \int \mathrm{d}^{4} x\left(\frac{\kappa}{2} T^{\mu \nu} h_{\mu \nu}+\frac{\kappa^{2}}{4} U^{\mu \nu \alpha \beta} h_{\mu \nu} h_{\alpha \beta}\right) \\
& +O\left(\kappa^{3}\right),
\end{aligned}
$$

where $h=h_{\mu}^{\mu}, T_{\mu \nu}$ is the stress tensor of the scalar field,

$$
T_{\mu \nu}=\partial_{\mu} \phi \partial_{\nu} \phi-\frac{1}{2} \eta_{\mu \nu} \partial_{\alpha} \phi \partial^{\alpha} \phi-\frac{1}{2} \eta_{\mu \nu} m^{2} \phi^{2},
$$

and

$$
\begin{aligned}
U_{\mu \nu \alpha \beta}= & -2 \eta_{\nu \alpha} \partial_{\mu} \phi \partial_{\beta} \phi+\eta_{\mu \nu} \partial_{\alpha} \phi \partial_{\beta} \phi \\
& +\left(\frac{1}{2} \eta_{\mu \alpha} \eta_{\nu \beta}-\frac{1}{4} \eta_{\mu \nu} \eta_{\alpha \beta}\right)\left(\partial^{\sigma} \phi \partial_{\sigma} \phi+m^{2} \phi^{2}\right) .
\end{aligned}
$$

Indices are raised and lowered with the background metric $\eta_{\mu \nu}$. We have kept only the free terms in the action for the gravitons $S_{h}$ because we shall only compute the lowest-order corrections of the self-energy of the $\phi$ field.

To compute these corrections we need to introduce counterterms in order to cancel divergences. Since our system is nonrenormalizable, it has to be understood as an effective field theory, a low-energy approximation of a more fundamental theory at the Planck scale [36,51-53]. In order to compute to a given precision $E^{n} \kappa^{n}$, where $E$ is the energy of the process, one has to introduce all possible counterterms compatible with the symmetry whose coefficients are of order $\kappa^{n}$ at most. In our case the most general action for the counterterms for the scalar field action up to order $\kappa^{2}$ which is compatible with the Poincare symmetry is

$$
\begin{aligned}
S_{\text {count }}= & -\int \mathrm{d}^{4} x\left[\frac{1}{2}\left(m_{0}^{2}-m^{2}\right) \phi^{2}+\frac{1}{2}(Z-1)\left(\partial_{\mu} \phi \partial^{\mu} \phi\right.\right. \\
& \left.\left.+m^{2} \phi^{2}\right)+\frac{1}{4} \kappa^{2} C_{0}\left(\partial_{\mu} \partial^{\mu} \phi\right)^{2}\right]+O\left(\kappa^{4}\right),
\end{aligned}
$$

where $m_{0}=m+O\left(\kappa^{2}\right)$ is the bare mass, $Z=1+O\left(\kappa^{2}\right)$ is the field renormalization parameter, and $C_{0}=C+O(1)$ is a bare four-derivative coefficient. The finite coefficient $C$ is a priori unknown and constitutes an external input of the theory. The value of $C$ should be determined by experiments or by knowledge of the underlying more fundamental theory. On
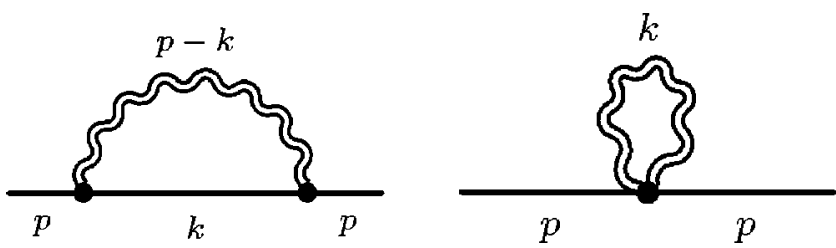

FIG. 1. The two Feynman diagrams needed for the calculation of the self-energy, respectively, $\Sigma_{(1)}(p)$ and $\Sigma_{(2)}(p)$.

the other hand, we do not introduce counterterms to the interaction action because we shall not compute vertex corrections.

Additionally the graviton action (3b) must be supplemented with a gauge-fixing term. We will work with the harmonic gauge $\partial_{\nu} h^{\mu \nu}-(1 / 2) \partial^{\mu} h=0$, whose appropriate gauge-fixing action is $[75,76]$

$$
S_{\mathrm{gf}}=-\int \mathrm{d}^{4} x\left(\partial_{\nu} h^{\mu \nu}-\frac{1}{2} \partial^{\mu} h\right)\left(\partial^{\lambda} h_{\mu \lambda}-\frac{1}{2} \partial_{\mu} h\right) .
$$

No Faddeev-Popov ghost fields are needed since we will not consider graviton self-interactions.

\section{ZERO TEMPERATURE}

The aim in this section is to compute the leading contribution to the vacuum self-energy of the scalar particle. At zero temperature the self-energy $\Sigma^{(T=0)}\left(p^{2}\right)$ is related to the Feynman propagator $G_{\mathrm{F}}^{(T=0)}(p)$ through

$$
G_{\mathrm{F}}^{(T=0)}(p)=\frac{-i}{p^{2}+m^{2}+\Sigma^{(T=0)}\left(p^{2}\right)}
$$

We recall that the self-energy can be computed as the sum of all one-particle irreducible diagrams with amputated external legs $[35,36,77]$. In order to regulate divergences appearing in the calculation we will use dimensional regularization $[78,79]$.

The two diagrams which may contribute to order $\kappa^{2}$ are shown in Fig. 1. One must also take into account the contribution of the counterterms:

$$
\begin{aligned}
\Sigma^{(T=0)}\left(p^{2}\right)= & \left(m_{0}^{2}-m^{2}\right)+(Z-1)\left(p^{2}+m^{2}\right)+\kappa^{2} C_{0} p^{4} \\
& +\Sigma_{(1)}^{(T=0)}\left(p^{2}\right)+\Sigma_{(2)}^{(T=0)}\left(p^{2}\right)+O\left(\kappa^{4}\right) .
\end{aligned}
$$

We first concentrate on the first diagram $\Sigma_{(1)}^{(T=0)}\left(p^{2}\right)$. Applying the Feynman rules described in Appendix A we find, in $d$ spacetime dimensions,

$$
\begin{aligned}
-i \Sigma_{(1)}^{(T=0)}\left(p^{2}\right)= & \mu^{\varepsilon} \int \frac{\mathrm{d}^{d} k}{(2 \pi)^{d}} \tau_{\mu \nu}(p, k) \tau_{\alpha \beta}(k, p) \\
& \times \frac{-i \mathcal{P}^{\mu \nu \alpha \beta}}{(p-k)^{2}-i \epsilon} \frac{-i}{k^{2}+m^{2}-i \epsilon},
\end{aligned}
$$


where $\varepsilon=4-d, \mathcal{P}_{\mu \nu \alpha \beta}$ and $\tau_{\mu \nu}(p, k)$ are given in Appendix $\mathrm{A}$, and $\mu$ is an arbitrary mass scale. Developing the products in the numerator we find

$$
\begin{aligned}
-i \Sigma_{(1)}^{(T=0)}\left(p^{2}\right)= & \frac{\kappa^{2}}{2} p^{2} \eta^{\mu \nu} I_{\mu \nu}(p)-\kappa^{2} m^{2} p^{\mu} I_{\mu}(p) \\
& -\kappa^{2} m^{4}(1+\varepsilon / 4) I(p)+O(\varepsilon),
\end{aligned}
$$

where the momentum integrals $I(p), I_{\mu}(p)$, and $I_{\mu \nu}(p)$ are defined and computed in Appendix B. The result for $\Sigma_{(1)}^{(T=0)}\left(p^{2}\right)$ is

$$
\begin{aligned}
\Sigma_{(1)}^{(T=0)}\left(p^{2}\right)= & \frac{\kappa^{2}}{(4 \pi)^{2}}\left[\frac{m^{4}}{\hat{\varepsilon}}+2 m^{4}+\frac{m^{2} p^{2}}{\hat{\varepsilon}}+2 m^{2} p^{2}\right. \\
& -\left(\frac{m^{6}}{2 p^{2}}+\frac{m^{4}}{2}\right) \ln \left(1+\frac{p^{2}}{m^{2}}-i \epsilon\right) \\
& \left.-\left(m^{4}+m^{2} p^{2}\right) \ln \left(\frac{p^{2}+m^{2}}{\mu}-i \epsilon\right)\right]+O(\varepsilon),
\end{aligned}
$$

where $1 / \hat{\varepsilon}=2 / \varepsilon-\gamma+\ln 4 \pi$. The second diagram $\Sigma_{(2)}^{(T=0)}\left(p^{2}\right)$ is a massless tadpole, and these are identically zero in dimensional regularization [78].

Once we include the counterterms and take the limit $\varepsilon$ $\rightarrow 0$ the renormalized self-energy is found to be

$$
\begin{aligned}
\Sigma^{(T=0)}\left(p^{2}\right)= & -\frac{\kappa^{2}}{(4 \pi)^{2}}\left(\frac{m^{6}}{2 p^{2}}+\frac{m^{4}}{2}\right) \ln \left(1+\frac{p^{2}}{m^{2}}-i \epsilon\right) \\
& -\frac{\kappa^{2}}{(4 \pi)^{2}}\left(m^{4}+m^{2} p^{2}\right) \ln \left(\frac{p^{2}+m^{2}}{\mu^{2}}-i \epsilon\right) \\
& +C \kappa^{2}\left(p^{2}+m^{2}\right)^{2}+O\left(\kappa^{4}\right),
\end{aligned}
$$

where we have absorbed divergences in the following way:

$$
\begin{aligned}
& m_{0}^{2}=m^{2}-C \kappa^{2} m^{4}+O\left(\kappa^{4}\right), \\
& Z=1+2 C \kappa^{2} m^{2}-\frac{\kappa^{2} m^{2}}{(4 \pi)^{2}}\left(\frac{1}{\hat{\varepsilon}}+2\right)+O\left(\kappa^{4}\right), \\
& C_{0}=C+O\left(\kappa^{4}\right) .
\end{aligned}
$$

In order to relate the bare and the renormalized parameters we have imposed the on-shell renormalization condition

$$
\Sigma^{(T=0)}\left(-m^{2}\right)=0
$$

which fixes the position of the pole to be $m^{2}$. The complete specification of the on-shell renormalization scheme also involves fixing the residue of the pole, which implies an addi- tional condition on the derivative of the self-energy $[35,36]$. However this second condition is found to be singular due to the divergence of the logarithms when evaluated on shell. This kind of singularity is generic for theories with interacting massless particles because of the absence of well-defined asymptotic regions in the presence of long-range forces. For a discussion of this point in the case of QED see Ref. [35]. Anyway in the paper we are just interested position of the pole, which is always well defined.

Note that the only divergence arises from the field renormalization. In particular, the four-derivative coefficient $C$ does not get renormalized: there are no logarithmic corrections to the $p^{4}$ term in the self-energy (13), and $C$ coincides with its classical value $C_{0}$. Therefore it would have been consistent not to include the four-derivative counterterm and simply take $C=0$ from the beginning.

The self-energy develops a negative imaginary part for $-p^{2}>m^{2}$

$\operatorname{Im} \Sigma^{(T=0)}\left(p^{2}\right)=\theta\left(-p^{2}-m^{2}\right) \frac{\kappa^{2}}{16 \pi}\left(\frac{m^{6}}{2 p^{2}}+\frac{3 m^{4}}{2}+m^{2} p^{2}\right)$,

which, according to the optical theorem [77], accounts for the probability of a scalar particle with momentum $p$ to decay into an on-shell scalar and an on-shell graviton. Notice that on shell $\left(p^{2}=-m^{2}\right)$ the imaginary part of the selfenergy vanishes, because there is no phase space available for the spontaneous emission of a graviton.

\section{FINITE TEMPERATURE}

In this section we compute the thermal contribution to the self-energy working within the real-time approach to thermal field theory. In this approach, which is briefly explained in Appendix C, the number of degrees of freedom is doubled, and one has to consider four propagators organized in a $2 \times 2$ matrix $G_{a b}(p)$. The self-energy also becomes a matrix $\Sigma^{a b}(p)$. We will concentrate on the retarded propagator $G_{\mathrm{R}}(p)=G_{11}(p)-G_{12}(p)$ for the following reasons: First, it is the one that exhibits simple analytical properties at finite temperature (analyticity in the upper $p^{0}$ plane); second, as shown in Appendix $\mathrm{C}$, it is directly connected with the retarded self-energy $\Sigma_{\mathrm{R}}=\Sigma^{11}(p)+\Sigma^{12}(p)$ through

$$
G_{\mathrm{R}}(p)=\frac{-i}{p^{2}+m^{2}+\Sigma_{\mathrm{R}}(p)}
$$

and, finally, the position of its poles have well-defined interpretations in terms of energies and thermalization rates. Furthermore, the retarded propagator is the one that is naturally obtained from analytic continuation from the Euclidean propagator in the imaginary-time formalism. For more details we refer to Appendix $\mathrm{C}$ and to Refs. [54-56]. A quantum mechanical model where this point will be studied in detail will be presented elsewhere [80]. 


\section{A. Self-energy: Real part}

Let us proceed to the calculation of the real part of $\Sigma_{\mathrm{R}}(p)$. Since $\operatorname{Re} \Sigma_{\mathrm{R}}(p)=\operatorname{Re} \Sigma^{11}(p)$ [see Eq. (C19)], we shall compute the real part of $\Sigma^{11}(p)$ instead. We have to consider both diagrams sketched in Fig. 1, where now internal propagators are taken to be thermal and of type 11:

$$
\begin{aligned}
\Sigma^{11}(p)= & \left(m_{0}^{2}-m^{2}\right)+(Z-1)\left(p^{2}+m^{2}\right)+\kappa^{2} C_{0} p^{4}+\Sigma_{(1)}^{11}(p) \\
& +\Sigma_{(2)}^{11}(p)+O\left(\kappa^{4}\right) .
\end{aligned}
$$

The first diagram $\Sigma_{(1)}^{11}(p)$ is given by

$$
\begin{aligned}
\Sigma_{(1)}^{11}(p)= & \frac{i \kappa^{2}}{2} \mu^{\varepsilon} \int \frac{\mathrm{d}^{d} k}{(2 \pi)^{d}}\left[\frac{1}{(p-k)^{2}-i \epsilon}\right. \\
& \left.+2 \pi i n\left(\left|p^{0}-k^{0}\right|\right) \delta\left((p-k)^{2}\right)\right]\left[\frac{1}{k^{2}+m^{2}-i \epsilon}\right. \\
& \left.+2 \pi i n\left(\left|k^{0}\right|\right) \delta\left(k^{2}-m^{2}\right)\right] g_{1}\left(p^{2}, k^{2}, p \cdot k\right),
\end{aligned}
$$

where

$$
\begin{aligned}
g_{1}\left(p^{2}, k^{2}, p \cdot k\right) & =-\frac{2}{\kappa^{2}} \tau_{\mu \nu}(p, k) \tau_{\alpha \beta}(k, p) \mathcal{P}^{\mu \nu \alpha \beta} \\
& =k^{2} p^{2}-2(k \cdot p) m^{2}-2(1+\varepsilon / 4) m^{4},
\end{aligned}
$$

the function $n(E)$ is the Bose-Einstein distribution,

$$
n(E)=\frac{1}{1-e^{E / T}},
$$

and we recall that $\varepsilon=4-d$ and that $\tau_{\mu \nu}(p, k)$ and $\mathcal{P}^{\mu \nu \alpha \beta}$ are given in Appendix A. On the other hand, at finite temperature the tadpole diagram $\Sigma_{(2)}^{11}(p)$ no longer vanishes since its temperature-dependent part gives a finite contribution,

$$
\Sigma_{(2)}^{11}(p)=-\frac{\kappa^{2}}{2} g_{3}\left(p^{2}\right) \int \frac{\mathrm{d}^{4} k}{(2 \pi)^{4}} 2 \pi \delta\left(k^{2}\right) n\left(\left|k^{0}\right|\right),
$$

where

$$
g_{3}\left(p^{2}\right)=-\frac{2 i}{\kappa^{2}} V_{\mu \nu \alpha \beta}(p, p) \mathcal{P}^{\mu \nu \alpha \beta}=10 m^{2}+4 p^{2}
$$

with $V_{\mu \nu \alpha \beta}(p, k)$ given in Appendix A.

To order $\kappa^{2}$ one has

$$
\begin{aligned}
\operatorname{Re} \Sigma^{11}(p)= & \operatorname{Re} \Sigma^{(T=0)}\left(p^{2}\right)-\frac{\kappa^{2}}{2}\left[A(p)+B(p)+C\left(p^{2}\right)\right] \\
& +O\left(\kappa^{4}\right)
\end{aligned}
$$

where $\Sigma^{(T=0)}\left(p^{2}\right)$ is the $T=0$ self-energy [see Eqs. (9) and (13)] and where the integrals $A(p), B(p)$, and $C\left(p^{2}\right)$ are defined through

$$
\begin{aligned}
A(p)= & \int \frac{\mathrm{d}^{4} k}{(2 \pi)^{3}} n\left(\left|p^{0}-k^{0}\right|\right) \delta\left((p-k)^{2}\right) \\
& \times g_{1}\left(p^{2}, k^{2}, p \cdot k\right) \mathrm{P} \frac{1}{k^{2}+m^{2}}, \\
B(p)= & \int \frac{\mathrm{d}^{4} k}{(2 \pi)^{3}} n\left(\left|k^{0}\right|\right) \delta\left(k^{2}+m^{2}\right) \\
& \times g_{1}\left(p^{2}, k^{2}, p \cdot k\right) \mathrm{P} \frac{1}{(p-k)^{2}},
\end{aligned}
$$

$$
C\left(p^{2}\right)=g_{3}\left(p^{2}\right) \int \frac{\mathrm{d}^{4} k}{(2 \pi)^{3}} n\left(\left|k^{0}\right|\right) \delta\left(k^{2}\right),
$$

with "P" meaning principal value. We have set $d=4$ because thermal contributions are ultraviolet finite. Note that the same renormalization process which makes the $T=0$ self-energy finite also renormalizes the $T>0$ self-energy; there is no need to introduce additional temperaturedependent counterterms. Hereafter we will concentrate in the on-shell results $p^{0}=E_{\mathbf{p}}=\sqrt{m^{2}+|\mathbf{p}|^{2}}$, since these are the ones we will need afterwards for the calculation of the thermal mass and the modified dispersion relation.

The integrals $A(p)$ and $C\left(p^{2}\right)$ take into account the effect of the thermal gravitons on the scalar particle. In the on-shell case it is possible to give an explicit expression for these integrals valid at any temperature (the details of the calculation can be found in Appendix D):

$$
A\left(E_{\mathbf{p}}, \mathbf{p}\right)=-\frac{1}{6} m^{2} T^{2}
$$

$$
C\left(-m^{2}\right)=\frac{1}{2} m^{2} T^{2}
$$

Notice that $A\left(E_{\mathbf{p}}, \mathbf{p}\right)$ does not depend on the threemomentum p. This is a surprise since Eq. (25a) is not manifestly Lorentz invariant. This simplification, which is also 
found in electrodynamics [37], will have important consequences when computing the modified dispersion relation.

The integral $B(p)$ takes into account the effect of the thermal scalars in the heat bath. For temperatures well below the mass $m$ there are almost no scalar particles in the bath, and this indeed shows up in an exponential suppression of $B\left(E_{\mathbf{p}}, \mathbf{p}\right)$ at low temperatures $T \ll m$ :

$$
B\left(E_{\mathbf{p}}, \mathbf{p}\right) \approx \sqrt{\frac{m^{5} T^{3}}{2 \pi^{3}}}\left(\frac{m^{2}+2|\mathbf{p}|^{2}}{3 m^{2}+4|\mathbf{p}|^{2}}\right) e^{-m / T}, \quad T \ll m .
$$

For temperatures of the order of the mass $m$, there are thermal scalar particles in the bath and they give a significant contribution to the self-energy. At high temperatures $T \gg m$, the leading contribution to the integral is given by

$$
\begin{aligned}
B\left(E_{\mathbf{p}}, \mathbf{p}\right) \approx & \frac{m^{2} T^{2} \sqrt{m^{2}+|\mathbf{p}|^{2}}}{24|\mathbf{p}|} \ln \left(\frac{2 \sqrt{m^{2}+|\mathbf{p}|^{2}}-|\mathbf{p}|}{2 \sqrt{m^{2}+|\mathbf{p}|^{2}}+|\mathbf{p}|}\right) \\
& +\frac{1}{8} m^{2} T^{2}, \quad T \gg m .
\end{aligned}
$$

The details of the calculation for both the low- and hightemperature limits are given in Appendix D.

Summarizing, according to Eqs. (24) and (26)-(29) the real part of the on-shell self-energy in the low- and hightemperature regimes is given by

$$
\operatorname{Re} \Sigma_{\mathrm{R}}\left(E_{\mathbf{p}}, \mathbf{p}\right) \approx \begin{cases}-\frac{1}{6} \kappa^{2} m^{2} T^{2}-\sqrt{\frac{m^{5} T^{3}}{8 \pi^{3}} \kappa^{2} e^{-m / T}\left(\frac{m^{2}+2|\mathbf{p}|^{2}}{3 m^{2}+4|\mathbf{p}|^{2}}\right),} & T \ll m, \\ \frac{1}{48} \kappa^{2} m^{2} T^{2}\left[-11+\frac{\sqrt{m^{2}+|\mathbf{p}|^{2}}}{|\mathbf{p}|} \ln \left(\frac{2 \sqrt{m^{2}+|\mathbf{p}|^{2}}+|\mathbf{p}|}{2 \sqrt{m^{2}+|\mathbf{p}|^{2}}-|\mathbf{p}|}\right)\right], & T \gg m .\end{cases}
$$

Notice that from the high-temperature result we can deduce that for massless particles the on-shell self-energy is exactly zero.

\section{B. Self-energy: Imaginary part}

Now we want to compute $\operatorname{Im} \Sigma_{\mathrm{R}}(p)$. Similarly to the previous subsection we could compute $\Sigma^{11}(p)$ and then make use of Eq. (C19); however, it is somewhat easier for us to compute $\Sigma^{12}(p)$ and $\Sigma^{21}(p)$ and make use of relation $(\mathrm{C} 18)$ instead. The self-energy $\Sigma^{12}(p)$ is given by

$$
\begin{aligned}
\Sigma^{12}(p)= & i \frac{\kappa^{2}}{2} \int \frac{\mathrm{d}^{4} k}{(2 \pi)^{2}} n\left(k^{0}\right) \operatorname{sgn}\left(k^{0}\right) n\left(p^{0}-k^{0}\right) \\
& \times \operatorname{sgn}\left(p^{0}-k^{0}\right) \delta\left((p-k)^{2}\right) \delta\left(k^{2}+m^{2}\right) \\
& \times g_{1}\left(p^{2}, k^{2}, p \cdot k\right),
\end{aligned}
$$

where we used the property $\theta\left(-p^{0}\right)+n\left(\left|p^{0}\right|\right)$ $=\operatorname{sgn}\left(p^{0}\right) n\left(p^{0}\right)$. In a similar way we find, for $\Sigma^{21}$,

$$
\begin{aligned}
\Sigma^{21}(p)= & i \frac{\kappa^{2}}{2} \int \frac{\mathrm{d}^{4} k}{(2 \pi)^{2}}\left[1+n\left(k^{0}\right)\right] \operatorname{sgn}\left(k^{0}\right) \\
& \times\left[1+n\left(p^{0}-k^{0}\right)\right] \operatorname{sgn}\left(p^{0}-k^{0}\right) \delta\left((p-k)^{2}\right) \\
& \times \delta\left(k^{2}+m^{2}\right) g_{1}\left(p^{2}, k^{2}, p \cdot k\right),
\end{aligned}
$$

where now we used $\theta\left(p^{0}\right)+n\left(\left|p^{0}\right|\right)=\operatorname{sgn}\left(p^{0}\right)\left[1+n\left(p^{0}\right)\right]$. Thus from Eq. (C18) and the two previous equations we get

$$
\operatorname{Im} \Sigma_{\mathrm{R}}(p)=-\frac{\kappa^{2}}{4} D(p)
$$

where $D(p)$ is defined by

$$
\begin{aligned}
D(p)= & \int \frac{\mathrm{d}^{4} k}{(2 \pi)^{2}} F\left(p^{0}, k^{0}\right) g_{1}\left(p^{2}, k^{2}, p \cdot k\right) \\
& \times \delta\left((p-k)^{2}\right) \delta\left(k^{2}+m^{2}\right),
\end{aligned}
$$

with

$F\left(p^{0}, k^{0}\right)=\operatorname{sgn}\left(k^{0}\right) \operatorname{sgn}\left(p^{0}-k^{0}\right)\left[1+n\left(k^{0}\right)+n\left(p^{0}-k^{0}\right)\right]$,

which can be developed to give

$$
F\left(p^{0}, k^{0}\right)=\frac{1}{2} \sinh \left(\frac{p^{0}}{2 T}\right) \operatorname{csch}\left(\frac{\left|p^{0}-k^{0}\right|}{2 T}\right) \operatorname{csch}\left(\frac{\left|k^{0}\right|}{2 T}\right) .
$$

After manipulating the integral $D(p)$ (the details can be found in Appendix D) the imaginary part of the self-energy can be expressed as the following phase-space integral:

$$
\operatorname{Im} \Sigma_{\mathrm{R}}(p)=\frac{\kappa^{2} m^{2}\left(m^{2}+2 p^{2}\right)}{32 \pi|\mathbf{p}|}\left|\int_{Q_{1}}^{Q_{2}} \mathrm{~d} Q F\left(p^{0}, Q\right)\right| .
$$

Let us now evaluate the integral in Eq. (37) for an arbitrary temperature. For simplicity, we restrict ourselves to the case $p^{0}>|\mathbf{p}|$, but including both $\left(p^{0}\right)^{2}>m^{2}+|\mathbf{p}|^{2}$ and $\left(p^{0}\right)^{2}<m^{2}+|\mathbf{p}|^{2}$. In this situation the integral can be performed analytically to give 


$$
\operatorname{Im} \Sigma_{\mathrm{R}}(p)=\frac{\kappa^{2} T m^{2}\left(m^{2}+2 p^{2}\right)}{32 \pi|\mathbf{p}|} \ln \left[\frac{\sinh \left(\frac{\left(p^{0}+|\mathbf{p}|\right)^{2}+m^{2}}{4 T\left(p^{0}+|\mathbf{p}|\right)}\right) \sinh \left(\frac{\left(p^{0}\right)^{2}-|\mathbf{p}|^{2}-m^{2}}{4 T\left(p^{0}-|\mathbf{p}|\right)}\right)}{\sinh \left(\frac{\left(p^{0}-|\mathbf{p}|\right)^{2}+m^{2}}{4 T\left(p^{0}-|\mathbf{p}|\right)}\right) \sinh \left(\frac{\left(p^{0}\right)^{2}-|\mathbf{p}|^{2}-m^{2}}{4 T\left(p^{0}+|\mathbf{p}|\right)}\right)}\right] .
$$

Making use of the property

$$
\ln (\sinh x) \approx|x|-\ln 2-i \pi \theta(-x)-e^{-2|x|}, \quad|x| \gg 1,
$$

we easily obtain the low-temperature approximation

$$
\begin{aligned}
\operatorname{Im} \Sigma_{\mathrm{R}}(p)= & \theta\left(-p^{2}-m^{2}\right) \frac{\kappa^{2}}{16 \pi}\left(\frac{m^{6}}{2 p^{2}}+\frac{3 m^{4}}{2}+m^{2} p^{2}\right) \\
& +O\left(e^{-m / T}\right),
\end{aligned}
$$

which is in agreement with the zero-temperature result of Eq. (16). At zero temperature the imaginary part of the selfenergy gives a net decay rate, but at finite temperature it is related to the rate $\Gamma$ at which a particle approaches thermal equilibrium [38]:

$$
\Gamma=\Gamma_{\mathrm{d}}-\Gamma_{\mathrm{c}}=-\frac{1}{p^{0}} \operatorname{Im} \Sigma_{\mathrm{R}} .
$$

In this expression $\Gamma_{\mathrm{d}}$ and $\Gamma_{\mathrm{c}}$ are, respectively, the annihilation and creation rates of particles with energy $p^{0}$, which take into account stimulated absorbtion and emission by the thermal bath. An arbitrary ensemble of particles with distribution function $f(E, t)$ approaches thermal equilibrium through

$$
f(E, t)=\frac{1}{e^{E / T}-1}+c(E) e^{-\Gamma(E) t},
$$

where $c(E)$ depends on the initial conditions. Hence the imaginary part of the self-energy corresponds to an observable quantity.

To order $\kappa^{2}$ a real particle can neither emit nor absorb a real graviton, because these processes are kinematically forbidden. Hence, at this order, the imaginary part of the selfenergy must vanish on shell. However in the on-shell limit $p^{0} \rightarrow E_{\mathbf{p}}=\sqrt{|\mathbf{p}|^{2}+m^{2}}$, we obtain, from Eq. (38),

$$
\operatorname{Im} \Sigma_{\mathrm{R}}(p) \underset{p^{0} \rightarrow E_{\mathbf{p}}}{\longrightarrow}-\frac{\kappa^{2} m^{4} T}{32 \pi|\mathbf{p}|} \ln \left(\frac{\sqrt{m^{2}+|\mathbf{p}|^{2}}+|\mathbf{p}|}{\sqrt{m^{2}+|\mathbf{p}|^{2}}-|\mathbf{p}|}\right),
$$

which is nonzero if $T \neq 0$.

This nonzero result for the on-shell self-energy is an artifact of not having introduced an infrared regularization; see Refs. [41,81]. To illustrate this point one can take the onshell limit directly in Eq. (37): in this limit $Q_{1}, Q_{2} \rightarrow 0$, so that the phase space of the integral vanishes while the ther- mal function $F\left(p^{0}, 0\right)$ diverges. Hence the nonvanishing result we obtained is a direct consequence of the infrared divergence of the Bose-Einstein distribution. When regulating the infrared behavior-for instance, by giving a tiny mass to the graviton-no imaginary part is found at $p^{2}=-m^{2}$. Additionally, had we worked in an arbitrary gauge we could have verified that the result of Eq. (42) is not even gauge invariant $[41,81]^{1}$

\section{DISCUSSION OF THE RESULTS}

At zero temperature, the position of the pole of the propagator gives the energy of the state and hence defines the dispersion relation, according to the Källen-Lehmann specral representation $[35,36,77]$. The position of the pole is found to be

$$
\left(p^{0}\right)^{2}=m^{2}+|\mathbf{p}|^{2}+\Sigma^{(T=0)}\left(-m^{2}\right)=m^{2}+|\mathbf{p}|^{2},
$$

where the second equality is a consequence of the renormalization condition (15). The dispersion relation is clearly Lorentz invariant, as expected.

Similarly, at finite temperature it can be shown [37] that the "effective" dispersion relation of the particle, given by the location of the poles of the retarded propagator, determines the inertial properties of the particle. The location of the poles is given by

$$
\left(p^{0}\right)^{2}-|\mathbf{p}|^{2}=m^{2}+\operatorname{Re} \Sigma_{R}\left(p^{0}, \mathbf{p}\right) .
$$

The thermal mass is obtained by setting $\mathbf{p}=\mathbf{0}$ :

$$
m_{T}^{2}=m^{2}+\operatorname{Re} \Sigma_{R}\left(m_{T}, \mathbf{0}\right) .
$$

In a Lorentz-invariant situation one would simply have $\left(p^{0}\right)^{2}=m_{T}^{2}+|\mathbf{p}|^{2}$, but in general there can be additional dependence on the three-momentum $\mathbf{p}$ on the right-hand side:

$$
\left(p^{0}\right)^{2}=m_{T}^{2}+|\mathbf{p}|^{2}+\mathcal{F}\left(\kappa, T, m_{T}, \mathbf{p}\right) .
$$

The Lorentz-breaking additional term in the dispersion relation leads to modifications of the group velocity of the particles $\mathbf{v}=\mathrm{d} p^{0} / \mathrm{d} \mathbf{p}$ :

$$
\mathbf{v}=\frac{\mathbf{p}}{p^{0}}+\frac{1}{2 p^{0}} \frac{\partial \mathcal{F}}{\partial \mathbf{p}}
$$

\footnotetext{
${ }^{1}$ We are grateful to A. Weldon for drawing our attention to this point.
} 
Let us find the explicit form of the thermal mass and Lorentz-breaking terms in both the low- and hightemperature regimes. Equation (44) can be solved perturbatively:

$$
\left(p^{0}\right)^{2}=m^{2}+|\mathbf{p}|^{2}+\operatorname{Re} \Sigma_{\mathrm{R}}\left(E_{\mathbf{p}}, \mathbf{p}\right)+O\left(\kappa^{4}\right),
$$

where we recall that $E_{\mathbf{p}}=\sqrt{m^{2}+|\mathbf{p}|^{2}}$. At low temperatures the modified dispersion relation, according to Eqs. (30) and (48), is approximately given by

$$
\left(p^{0}\right)^{2} \approx m_{T}^{2}+|\mathbf{p}|^{2}-\kappa^{2} \sqrt{\frac{m^{5} T^{3}}{2 \pi^{3}}} e^{-m / T}\left(\frac{|\mathbf{p}|^{2}}{3 m^{2}+4|\mathbf{p}|^{2}}\right),
$$

where the leading contribution to the thermal mass is

$$
m_{T}^{2} \approx m^{2}-\frac{1}{6} \kappa^{2} m^{2} T^{2} .
$$

In the high-three-momentum limit the term which modifies the dispersion relation becomes a constant and can be reabsorbed in the thermal mass. The group velocity is modified according to Eq. (47):

$$
\mathbf{v} \approx \frac{\mathbf{p}}{p^{0}}\left(1-\frac{\kappa^{2} m^{9 / 2} T^{3 / 2} e^{-m / T}}{\sqrt{2 \pi^{3}}\left(3 m^{2}+4|\mathbf{p}|^{2}\right)^{2}}\right) .
$$

Notice that at low temperatures the Lorentz-breaking term carries a Boltzmann factor $e^{-m / T}$. This is due to the mentioned fact that the nontrivial momentum dependence comes from the thermal scalar particles whose abundance is exponentially suppressed at low temperatures. Analogously to what happens in electrodynamics [37], the effect of the graviton bath only shows up in the thermal mass.

At high temperature $T \gg m$, the modified dispersion relation is found to be

$$
\left(p^{0}\right)^{2} \approx m_{T}^{2}+|\mathbf{p}|^{2}+\frac{\kappa^{2} m^{2} T^{2}}{48}\left[\frac{E_{\mathbf{p}}}{|\mathbf{p}|} \ln \left(\frac{2 E_{\mathbf{p}}+|\mathbf{p}|}{2 E_{\mathbf{p}}-|\mathbf{p}|}\right)-1\right],
$$

and the thermal mass is

$$
m_{T}^{2} \approx m^{2}-\frac{11}{48} \kappa^{2} m^{2} T^{2} .
$$

At high three-momentum the modification of the dispersion relation can be also reabsorbed in the thermal mass. The group velocity is given by

$$
\begin{array}{r}
\mathbf{v} \approx \frac{\mathbf{p}}{p^{0}}\left[1+\frac{1}{96} \frac{\kappa^{2} T^{2} m^{4}}{\left(4 m^{2}+3|\mathbf{p}|^{2}\right)|\mathbf{p}|^{2}}\right. \\
\left.-\frac{1}{96} \frac{\kappa^{2} T^{2} m^{4}}{|\mathbf{p}|^{3} E_{\mathbf{p}}} \ln \left(\frac{2 E_{\mathbf{p}}+|\mathbf{p}|}{2 E_{\mathbf{p}}-|\mathbf{p}|}\right)\right] .
\end{array}
$$

At high temperature the terms which break the Lorentz symmetry are no longer exponentially suppressed. Notice also that Eq. (52) shows that there is no modification to the dispersion relation for massless scalars.

The Lorentz-breaking term is negative in the lowtemperature case, which implies that the speed of propagation is lowered with respect to the standard relativistic case. In contrast, the Lorentz-breaking corrections have a positive sign at high temperatures, so that the speed of propagation is increased. However, it is always lower than the speed of light, as can be seen by expanding Eq. (54) in the ultrarelativistic limit:

$$
\mathbf{v} \approx \frac{\mathbf{p}}{|\mathbf{p}|}\left(1-\frac{m^{2}\left[48+\kappa^{2} T^{2}(\ln 3-1)\right]}{96|\mathbf{p}|^{2}}+\cdots\right) .
$$

We should emphasize that the above breaking of the Lorentz symmetry is only effective: if one applies a Lorentz transformation simultaneously to the particle and bath all results are Lorentz invariant. This can be seen can by introducing the unit vector $l^{\mu}$ which gives the four-velocity of the thermal bath. Then the energy and three-momentum of the particle with respect to the bath are

$$
p^{0}=-l_{\mu} p^{\mu}, \quad|\mathbf{p}|=\sqrt{\left(l_{\mu} p^{\mu}\right)^{2}+p_{\mu} p^{\mu}} .
$$

In this article for obvious reasons of simplicity we worked in the bath rest frame where $l^{\mu}=(1,0,0,0)$.

For low values of the momentum the effective dispersion relation can be expanded in powers of $\mathbf{p}$. For instance, in the low-temperature case, for momenta satisfying $|\mathbf{p}|<m$, the effective dispersion relation (49) can be expanded as

$$
\begin{aligned}
\left(p^{0}\right)^{2} \approx & m_{T}^{2}+|\mathbf{p}|^{2}-\frac{\kappa^{2} m^{5 / 2} T^{3 / 2} e^{-m / T}}{\sqrt{2 \pi^{3}}} \frac{|\mathbf{p}|^{2}}{3 m^{2}} \\
& +\frac{\kappa^{2} m^{5 / 2} T^{3 / 2} e^{-m / T}}{\sqrt{2 \pi^{3}}} \frac{2|\mathbf{p}|^{4}}{27 m^{4}}+\cdots
\end{aligned}
$$

It is worth mentioning the difference between this expansion and that of Eq. (1). In a fundamental approach to the Lorentz symmetry breaking one expects each additional power of momentum to be suppressed by increasing powers of the Planck mass, whereas in the effective breaking approach we have pursued there are several energy scales. Hence there is more freedom in the possible values of the suppression factor. For instance, in the low-temperature regime-i.e., in Eq. (57) - the $|\mathbf{p}|^{4}$ term is suppressed by $(T / m)^{3 / 2} e^{-m / T} \ll 1$ in Planck units. The fact that in the latter approach there are more energy scales also explains why modifications to the $|\mathbf{p}|^{2}$ term are present in Eq. (57) while they are not included in Eq. (1). Notice also that the corrections that we found only contain even powers of the momentum and thus are in agreement with the results of Ref. [82].

In the present universe the effects we discussed are completely negligible when applied to electrons or protons, both because they are proportional to the Planck length square and because they are exponentially suppressed. To obtain relevant effects, one should consider Planckian temperatures. However, in this case perturbation theory around a flat- 

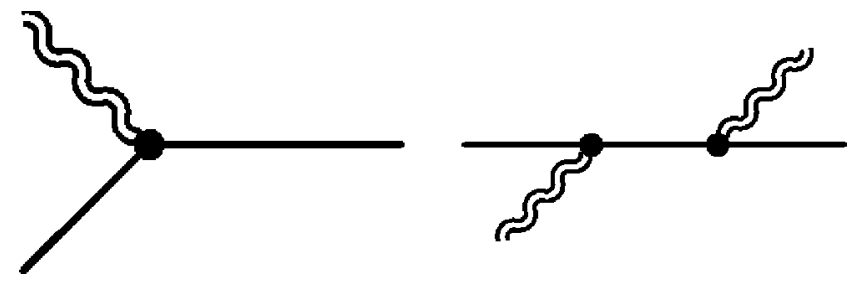

FIG. 2. Diagrams such as the one depicted on the left would contribute to order $\kappa^{2}$ to the thermalization rate at finite temperature and hence to the imaginary part of the self-energy. However, all those one-vertex diagrams are identically zero because of kinematical reasons. The first nonvanishing contribution would be due to Compton-like diagrams such as the one depicted on the right, and hence it would be a $\kappa^{4}$ contribution.

spacetime approximation probably fails. Therefore the results obtained here should be considered an indication that effective violations of Lorentz invariance indeed occur and that gravitational interactions cannot be neglected when exploring highly energetic regions of dispersion relations.

Whereas the real part of the self-energy gives the change in energy of the particle and hence the dispersion relation, the imaginary part accounts for the dissipative effects and gives the thermalization rate. As we have already commented, at the order $\kappa^{2}$ the imaginary part of the self-energy is zero. We do not see the thermalization effect since all processes which could contribute to it, such as the one shown in Fig. 2 (on the left), are kinematically forbidden. In order to account for thermalization effects we should compute the imaginary part of the self-energy to order $\kappa^{4}$. At this order, the particle can exchange momentum with the thermal bath through processes such as the one to the right of Fig. 2, which would correspond to the Compton scattering in electrodynamics. Notice here a significant difference with respect to the vacuum case: while at zero temperature the self-energy is purely real to all orders of perturbation theory (because the particle is stable), at finite temperature it will acquire an imaginary contribution to order $\kappa^{4}$.

Let us end with a short summary of the main points of the paper. We have illustrated with a particular example how local Lorentz symmetry is effectively violated because of the interactions with a nontrivial ensemble of metric fluctuations, even if Lorentz symmetry holds at a fundamental level. We have also shown how this effective violation can be addressed at low energies within standard physics. The main quantitative result of the paper are Eqs. (49) and (52), which explicitly show the modifications of the dispersion relation. As in the electromagnetic case, this effect is exponentially suppressed at low temperatures. Moreover, the modifications of the dispersion relation are suppressed when the threemomentum of the massive particle (defined in the heat bath rest frame) is much larger than the temperature and mass. This last result is somewhat unexpected since the gravitational coupling grows with the energy, unlike the electromagnetic case. Therefore, in spite of the derivative coupling, no violation of Lorenz invariance is found in the highmomentum limit, at least at one loop. Finally we have also shown that gravitational interactions do not generate a fourderivative term in the scalar field action.
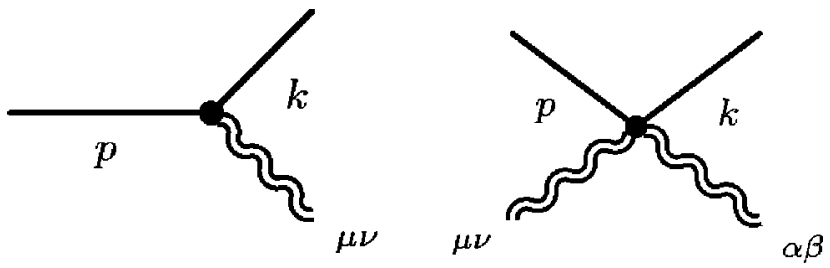

FIG. 3. On the left, the two-scalar-one-graviton vertex, denoted by $\tau_{\mu \nu}(p)$. On the right, the two-scalar-two-graviton vertex, denoted by $V_{\mu \nu \alpha \beta}(p)$.

\section{ACKNOWLEDGMENTS}

We would like to thank E. Calzetta, J. F. Donoghue, X. Garcia i Tormo, B.-L. Hu, A. Roura, and A. Weldon for their useful suggestions and for interesting discussions during various stages of this project. We are grateful to E. Gunzig for the organization of the Peyresq Physics meetings which stimulated discussions on this topic. D.A. acknowledges support of a FI grant from the Generalitat de Catalunya. This work is also partially supported by MICYT Research Project No. FPA-2001-3598 and European Project No. HPRN-CT2000-00131.

\section{APPENDIX A: FEYNMAN RULES}

At zero temperature the free propagator for the scalar field is

$$
G_{\mathrm{F}}^{(0, T=0)}(p)=\frac{-i}{p^{2}+m^{2}-i \epsilon},
$$

and the free propagator for the gravitons in the harmonic gauge is $[51,52,76]$

$$
\left(\Delta_{\mathrm{F}}\right)_{\mu \nu \alpha \beta}^{(T=0)}(p)=\frac{-i \mathcal{P}_{\mu \nu \alpha \beta}}{p^{2}-i \epsilon},
$$

where

$$
\mathcal{P}_{\mu \nu \alpha \beta}=\frac{1}{2}\left(\eta_{\mu \alpha} \eta_{\nu \beta}+\eta_{\mu \beta} \eta_{\nu \alpha}-\frac{2}{d-2} \eta_{\mu \nu} \eta_{\alpha \beta}\right)
$$

in $d$ spacetime dimensions. In four dimensions, $\mathcal{P}_{\mu \nu \alpha \beta}$ $=\left(\eta_{\mu \alpha} \eta_{\nu \beta}+\eta_{\mu \beta} \eta_{\nu \alpha}-\eta_{\mu \nu} \eta_{\alpha \beta}\right) / 2$.

The two-scalar-one-graviton vertex, shown in Fig. 3, is given by [52]

$$
\tau_{\mu \nu}(p, k)=\frac{i \kappa}{2}\left[p_{\mu} k_{\nu}+p_{\nu} k_{\mu}-(p \cdot k) \eta_{\mu \nu}-m^{2} \eta_{\mu \nu}\right]
$$

and the two-scalar-two-graviton vertex, also shown in Fig. 3 , is given by [52] 


$$
\begin{aligned}
V_{\mu \nu \alpha \beta}(p, k)= & -i \kappa^{2}\left[I_{\mu \nu \rho \lambda} I_{\sigma \alpha \beta}^{\lambda}\left(p^{\rho} k^{\sigma}+p^{\sigma} k^{\rho}\right)\right. \\
& +\frac{1}{2}\left(\eta_{\mu \nu} I_{\alpha \beta \rho \sigma}+\eta_{\alpha \beta} I_{\mu \nu \rho \sigma}\right) p^{\rho} k^{\sigma} \\
& \left.-\frac{1}{2}\left(I_{\mu \nu \alpha \beta}-\frac{1}{2} \eta_{\mu \nu} \eta_{\alpha \beta}\right)\left(p \cdot k+m^{2}\right)\right],
\end{aligned}
$$

where

$$
I_{\mu \nu \alpha \beta}=\frac{1}{2}\left(\eta_{\mu \alpha} \eta_{\nu \beta}+\eta_{\mu \beta} \eta_{\nu \alpha}\right)
$$

At finite temperature the free propagators for the scalar field are given by Eq. (C11) and the free propagators for the gravitons are $\left(\Delta_{a b}\right)_{\mu \nu \alpha \beta}(p)=\left.\mathcal{P}_{\mu \nu \alpha \beta} G_{a b}^{(0)}(p)\right|_{m=0}$. Vertices type 1 are described by $\tau_{\mu \nu}$ and $V_{\mu \nu \alpha \beta}$ and vertices type 2 are described by $-\tau_{\mu \nu}$ and $-V_{\mu \nu \alpha \beta}$.

\section{APPENDIX B: INTEGRALS IN DIMENSIONAL REGULARIZATION}

In Eq. (11) we need the integrals

$$
\begin{gathered}
I(p)=\mu^{\varepsilon} \int \frac{\mathrm{d}^{d} k}{(2 \pi)^{d}} \frac{1}{\left[k^{2}+m^{2}-i \epsilon\right]\left[(p-k)^{2}-i \epsilon\right]}, \\
I_{\mu}(p)=\mu^{\varepsilon} \int \frac{\mathrm{d}^{d} k}{(2 \pi)^{d}} \frac{k_{\mu}}{\left[k^{2}+m^{2}-i \epsilon\right]\left[(p-k)^{2}-i \epsilon\right]}, \\
I_{\mu \nu}(p)=\mu^{\varepsilon} \int \frac{\mathrm{d}^{d} k}{(2 \pi)^{d}} \frac{k_{\mu} k_{\nu}}{\left[k^{2}+m^{2}-i \epsilon\right]\left[(p-k)^{2}-i \epsilon\right]} .
\end{gathered}
$$

The result in arbitrary $d$ dimensions after series expansion in $\varepsilon=4-d$ is

$$
\begin{aligned}
I= & \frac{i}{(4 \pi)^{2}}\left[\frac{1}{\hat{\varepsilon}}+2-\frac{m^{2}}{p^{2}} \ln \left(1+\frac{p^{2}}{m^{2}}-i \epsilon\right)\right. \\
& \left.-\ln \left(\frac{p^{2}+m^{2}}{\mu^{2}}-i \epsilon\right)\right]+O(\varepsilon), \\
I_{\mu}(p)= & \frac{i p_{\mu}}{2(4 \pi)^{2}}\left[\frac{1}{\hat{\varepsilon}}+2-\frac{m^{2}}{p^{2}}+\frac{m^{4}}{p^{4}} \ln \left(1+\frac{p^{2}}{m^{2}}-i \epsilon\right)\right. \\
& \left.-\ln \left(\frac{p^{2}+m^{2}}{\mu^{2}}-i \epsilon\right)\right]+O(\varepsilon),
\end{aligned}
$$

$$
\begin{aligned}
I_{\mu \nu}(p)= & -\frac{\eta_{\mu \nu}}{2(4 \pi)^{2}}\left[\frac{m^{2}}{2 \hat{\varepsilon}}+\frac{p^{2}}{6 \hat{\varepsilon}}+\frac{m^{4}}{6 p^{2}}+\frac{7 m^{2}}{6}+\frac{4 p^{2}}{9}\right. \\
& -\left(\frac{m^{6}}{6 p^{4}}+\frac{m^{4}}{2 p^{2}}\right) \ln \left(1+\frac{p^{2}}{m^{2}}-i \epsilon\right)-\left(\frac{m^{2}}{2}+\frac{p^{2}}{6}\right) \\
& \left.\times \ln \left(\frac{p^{2}+m^{2}}{\mu^{2}}-i \epsilon\right)\right]+\frac{p_{\mu} p_{\nu}}{(4 \pi)^{2}}\left[\frac{1}{3 \hat{\varepsilon}}-\frac{m^{6}}{3 p^{6}}\right. \\
& \times \ln \left(1+\frac{p^{2}}{m^{2}}-i \epsilon\right)-\frac{1}{3} \ln \left(\frac{p^{2}+m^{2}}{\mu^{2}}-i \epsilon\right) \\
& \left.\times+\frac{m^{4}}{3 p^{4}}-\frac{m^{2}}{6 p^{2}}+\frac{13}{18}\right]+O(\varepsilon)
\end{aligned}
$$

where

$$
\frac{1}{\hat{\varepsilon}}=\frac{2}{\varepsilon}-\gamma+\ln 4 \pi
$$

\section{APPENDIX C: REAL-TIME THERMAL FIELD THEORY}

For the benefit of those readers who might be unfamiliar with the subject we give in this appendix a brief account of those aspects of real-time thermal field theory which are needed in the paper. For a complete introduction to the thermal field theory we address the reader to Refs. [54-56]; see also Ref. [83] for an approach similar to ours.

\section{Correlation functions}

In thermal field theory the initial state for the fields is assumed to be a thermal state at temperature $T=1 / \beta$, which is characterized by a density matrix

$$
\hat{\rho}=\frac{e^{-\beta \hat{H}}}{\operatorname{Tr}\left(e^{-\beta \hat{H}}\right)},
$$

where $\hat{H}$ is the Hamiltonian operator of the system (for the purposes of this appendix we shall consider a scalar field $\phi$ ). The generating functional $Z_{\mathcal{C}}[j]$ is defined as

$$
Z_{\mathcal{C}}[j]=\operatorname{Tr}\left[\hat{\rho} T_{\mathcal{C}} \exp \left(i \int_{\mathcal{C}} \mathrm{d} t \int \mathrm{d}^{3} \mathbf{x} \hat{\phi}(x) j(x)\right)\right],
$$

where $\hat{\phi}(x)$ is the field operator in the Heisenberg picture, $\mathcal{C}$ is a certain path in the complex $t$ plane, $T_{\mathcal{C}}$ means ordering along this path, and $j(x)$ is a classical external source. By functional differentiation of the generating functional with respect to $\phi$, path-ordered correlation functions can be obtained. The generating functional may be computed in a path-integral representation as

$$
Z_{\mathcal{C}}[j]=\int \mathcal{D} \phi \exp \left(i \int_{\mathcal{C}} \mathrm{d} t \int \mathrm{d}^{3} \mathbf{x}\{\mathcal{L}[\phi(x)]+j(x) \phi(x)\}\right),
$$




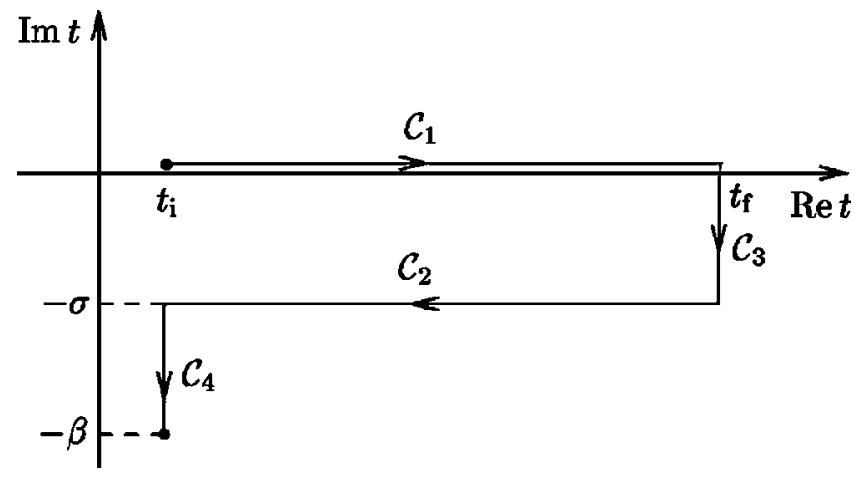

FIG. 4. Integration contour in the complex-time plane used in the real-time approach to thermal field theory. The choice $\sigma=0^{+}$ makes the formalism analogous to the CTP approach to field theory.

where $\mathcal{L}$ is the Lagrangian density and the contour $\mathcal{C}$ is now restricted to begin at some initial real time $t_{\mathrm{i}}$ and to end at $t_{\mathrm{i}}-i \beta$. The boundary conditions for the path integral are $\phi\left(t_{\mathrm{i}}, \mathbf{x}\right)=\phi\left(t_{\mathrm{i}}-i \beta, \mathbf{x}\right)$. Different elections for $\mathcal{C}$ lead to different approaches to thermal field theory: a straight line from $t_{\mathrm{i}}$ to $t_{\mathrm{i}}-i \beta$ leads to the imaginary-time formalism, and the contour shown in Fig. 4 leads to the real-time formalism. By choosing $\sigma=0^{+}$our formalism will coincide with the CTP approach to nonequilibrium field theory [57-59]. In fact many of the properties listed below are not limited to the thermal case but are valid in a more general nonequilibrium situation. We shall try to specify in each case which properties are general and which ones are particular to the thermal case.

If we are interested in real-time correlation functions, the path along $\mathcal{C}_{3}$ and $\mathcal{C}_{4}$ can be usually neglected once we properly take into account the boundary conditions of the path integral. If we define $\phi_{1,2}(t, \mathbf{x})=\phi(t, \mathbf{x})$ and $j_{1,2}(t, \mathbf{x})$ $=j(t, \mathbf{x})$ for $t \in \mathcal{C}_{1,2}$ and take $t_{\mathrm{i}} \rightarrow-\infty$ and $t_{\mathrm{f}} \rightarrow \infty$, the generating functional can be reexpressed as $Z_{\mathcal{C}}[j]=Z_{34} Z\left[j_{1}, j_{2}\right]$, where $Z_{34}$ represents the constant contribution from the segments $\mathcal{C}_{3}$ and $\mathcal{C}_{4}$ and $Z\left[j_{1}, j_{2}\right]$ is

$$
\begin{aligned}
Z\left[j_{1}, j_{2}\right]= & \int \mathcal{D} \phi_{1} \mathcal{D} \phi_{2} \exp \left(i \int \mathrm { d } ^ { 4 } x \left\{\mathcal{L}\left[\phi_{1}(x)\right]\right.\right. \\
& \left.\left.+j_{1}(x) \phi_{1}(x)\right\}\right) \exp \left(-i \int \mathrm{d}^{4} x\left\{\mathcal{L}\left[\phi_{2}(x)\right]\right.\right. \\
& \left.\left.+j_{2}(x) \phi_{2}(x)\right\}\right)
\end{aligned}
$$

Correlation functions are defined by second functional differentiation with respect to the external source $j(x)$ :

$$
G_{a b}\left(x, x^{\prime}\right)=\left.\frac{1}{Z[0,0]} \frac{\delta^{2} Z\left[j_{1}, j_{2}\right]}{\delta j^{a}(x) \delta j^{b}(x)}\right|_{j_{1}=j_{2}=0},
$$

where $a, b=1,2$. In operator language correlation functions can be written as

$$
G_{11}\left(x, x^{\prime}\right)=\left\langle T \hat{\phi}(x) \hat{\phi}\left(x^{\prime}\right)\right\rangle,
$$

$$
\begin{aligned}
& G_{12}\left(x, x^{\prime}\right)=\left\langle\hat{\phi}(x) \hat{\phi}\left(x^{\prime}\right)\right\rangle, \\
& G_{21}\left(x, x^{\prime}\right)=\left\langle\hat{\phi}\left(x^{\prime}\right) \hat{\phi}(x)\right\rangle, \\
& G_{22}\left(x, x^{\prime}\right)=\left\langle\widetilde{T} \hat{\phi}(x) \hat{\phi}\left(x^{\prime}\right)\right\rangle,
\end{aligned}
$$

where $(\widetilde{T}) T$ is the (anti-)time-ordering operator, and where the average means $\langle\cdots\rangle=\operatorname{Tr}(\hat{\rho} \cdots)$. Lowercase roman indices are raised and lowered with the "metric" $c_{a b}$ $=\operatorname{diag}(1,-1)$. From the above expressions it can be readily seen that not all correlation functions are independent. The following relations in Fourier space are a consequence of Eqs. (C6):

$$
\begin{gathered}
G_{11}(p)=G_{22}^{*}(p), \quad G_{12}(p)=G_{21}(-p), \\
G_{11}(p)+G_{22}(p)=G_{12}(p)+G_{21}(p),
\end{gathered}
$$

where correlation functions in Fourier space are

$$
G_{a b}(p)=\int \mathrm{d}^{4} x e^{-i p \cdot \Delta} G_{a b}(X+\Delta / 2, X-\Delta / 2),
$$

where we have introduced the new variables $\Delta=x-x^{\prime}$ and $X=\left(x+x^{\prime}\right) / 2 .^{2}$ Additionally, in the case of an initial thermal state the following relation, a consequence of the KuboMartin-Schwinger (KMS) formula (equivalent to the fluctuation-dissipation theorem, in another context), is also verified:

$$
G_{11}(p)+G_{22}(p)=e^{-\beta p^{0}} G_{12}(p)+e^{\beta p^{0}} G_{21}(p) .
$$

Thus, in thermal field theory knowledge of just one correlation function determines all of them.

An important correlation function is the retarded propagator, which is defined as

$$
G_{\mathrm{R}}\left(x, x^{\prime}\right)=\theta\left(x^{0}-x^{\prime 0}\right)\left\langle\left[\hat{\phi}(x), \hat{\phi}\left(x^{\prime}\right)\right]\right\rangle,
$$

and is related to the other correlation functions through

$$
G_{\mathrm{R}}(p)=G_{11}(p)-G_{12}(p) .
$$

The retarded propagator has the remarkable property that has well-defined analyticity properties at finite temperature, as opposite to most other propagators: it is analytic in the upper half of the complex $p^{0}$ plane. Furthermore, the retarded propagator is the one that one naturally obtains from an analytic continuation of the Euclidian propagator in the imaginary-time formalism [84].

\footnotetext{
${ }^{2}$ In order to avoid cumbersome notation the same symbol is used for correlators in configuration space and in Fourier space. Notice that Fourier-transformed propagators depend on $X$ if the initial state is nonhomogeneous. Since our primary concern here is thermal states, which are homogeneous, we do not indicate explicitly this $X$ dependence.
} 


\section{Perturbation theory}

Perturbation theory can be organized in a similar way as in the zero-temperature case, but taking into account that at finite temperature there are two kind of vertices (1 and 2) and four kind of propagators $(11,12,21$, and 22) which link the two vertices. Vertices type 2 carry an additional minus sign with respect to vertices type 1. When computing Feynman diagrams one has to sum over all possible internal vertices. For a real scalar field of mass $m$, free propagators are given by

$$
\left.\begin{array}{rl}
G_{a b}^{(0)}(p)= & 2 \pi \delta\left(p^{2}+m^{2}\right) \theta\left(-p^{0}\right) \\
2 \pi \delta\left(p^{2}+m^{2}\right) \theta\left(p^{0}\right) & \frac{i}{p^{2}+m^{2}+i \epsilon}
\end{array}\right)
$$

where $n(E)$ is the Bose-Einstein distribution function:

$$
n(E)=\frac{1}{1-e^{\beta E}}
$$

Thermal contributions to Feynman diagrams are always finite in the ultraviolet regime because the Bose-Einstein function acts as a soft cutoff for momenta larger than the temperature $T$. The counterterms which renormalize the theory at zero temperature also renormalize the theory at finite temperature. Note also that the thermal part of the propagator, which breaks the Lorentz symmetry through an explicit dependence on $p^{0}$, is always on shell.

Instead of working with the four propagators $G_{a b}(p)$ in the thermal case one can reorganize perturbation theory in a way such that just retarded and advanced propagators are involved [85]. In this case one has to consider how Feynman rules are transformed when working with the retarded and advanced basis.

\section{Self-energy}

In a thermal or, more generally, in a nonequilibrium situation, the self-energy has a matricial structure and is implicitly defined through the equation

$$
G_{a b}(p)=G_{a b}^{(0)}(p)+G_{a c}^{(0)}(p)\left[-i \Sigma^{c d}(p)\right] G_{d b}(p),
$$

where $G_{a b}^{(0)}(p)$ are the free propagators of the theory. The $a b$ component of the self-energy can be computed, similarly to the vacuum case, as the sum of all one-particle irreducible diagrams with amputated external legs that begin and end with type $a$ and type $b$ vertices, respectively.

In general the self-energy components verify the nonperturbative relations

$$
\Sigma^{11}(p)=-\left(\Sigma^{22}\right)^{*}(p), \quad \Sigma^{12}(p)=\Sigma^{21}(-p)
$$

$$
\Sigma^{11}(p)+\Sigma^{22}(p)=-\Sigma^{12}(p)-\Sigma^{21}(p)
$$

which can be obtained from Eqs. (C7) and (C13). The following equation is just verified if the initial state is thermal:

$$
\Sigma^{11}(p)+\Sigma^{22}(p)=-e^{-\beta p^{0}} \Sigma^{12}(p)-e^{\beta p^{0}} \Sigma^{21}(p) .
$$

Thus, all the components of the self-energy can be determined from knowledge of just one of them. Combining relations (C14) we obtain

$$
\operatorname{Im} \Sigma^{11}(p)=\frac{i}{2}\left[\Sigma^{12}(p)+\Sigma^{21}(p)\right] .
$$

This last equation can be directly obtained from the cutting rules at finite temperature.

A particularly useful combination is the retarded selfenergy, defined as $\Sigma_{\mathrm{R}}(p)=\Sigma^{11}(p)+\Sigma^{12}(p)$. It is related to the retarded propagator through

$$
G_{\mathrm{R}}(p)=\frac{-i}{p^{2}+m^{2}+\Sigma_{\mathrm{R}}(p)} .
$$

The above relation, which justifies the name of retarded selfenergy for $\Sigma_{\mathrm{R}}(p)$, can be demonstrated by expanding the matrix equation (C13) and using the relations (C7) and (C14). Similar relations hold for the advanced propagator $G_{\mathrm{A}}(p)=G_{\mathrm{R}}^{*}(p)$ and the advanced self-energy $\Sigma_{\mathrm{A}}(p)$ $=-\Sigma_{\mathrm{R}}^{*}(p)$. Notice that a diagonal relation such as Eq. (C17) can be found only for the retarded (or advanced) propagator.

According to Eq. (C16), the imaginary part of the retarded self-energy can be also expressed as

$$
\operatorname{Im} \Sigma_{\mathrm{R}}(p)=\frac{i}{2}\left[\Sigma^{21}(p)-\Sigma^{12}(p)\right]
$$

For a thermal state $\Sigma_{\mathrm{R}}(p)$ is related to $\Sigma^{11}(p)$ through

$$
\Sigma^{\mathrm{R}}(p)=\operatorname{Re} \Sigma^{11}(p)+\tanh \left(\frac{p^{0}}{2 T}\right) \operatorname{Im} \Sigma^{11}(p) .
$$

\section{APPENDIX D: COMPUTATION OF $A(p), B(p), C\left(p^{2}\right)$, AND $D(p)$}

In this appendix we shall compute the integrals $A(p)$, $B(p), C\left(p^{2}\right)$, and $D(p)$ which appear in the calculation of the self-energy at finite temperature; see Eqs. (25a)-(25c) and (34).

Let us start by computing the integral $A(p)$, defined in Eq. (25a). The Dirac delta can be expanded as

$$
\delta\left((p-k)^{2}\right)=\delta\left(q^{2}\right)=\frac{1}{2|\mathbf{q}|}\left[\delta\left(-q^{0}+|\mathbf{q}|\right)+\delta\left(q^{0}+|\mathbf{q}|\right)\right],
$$

where we have introduced the new variable $q=p-k$ and where $p=\left(p^{0}, \mathbf{p}\right)$ and $q=\left(q^{0}, \mathbf{q}\right)$. Introducing now spherical coordinates $(\phi, \theta)$ in the three spatial dimensions, with $\theta$ 
being the angle between $\mathbf{p}$ and $\mathbf{q}$, and integrating with respect to $q^{0}$ with the aid of the delta function we get

$$
\begin{aligned}
A(p)= & \int_{0}^{\infty} \frac{n(Q) Q \mathrm{~d} Q}{2(2 \pi)^{2}} \\
& \times \int_{-1}^{1} \mathrm{~d} x\left[\mathrm{P} \frac{g_{2}\left(p^{2}, 0,-p^{0} Q+P Q x\right)}{-\left(p^{0}\right)^{2}+P^{2}+2 p^{0} Q-2 P Q x+m^{2}}\right. \\
& \left.+\mathrm{P} \frac{g_{2}\left(p^{2}, 0, p^{0} Q+P Q x\right)}{-\left(p^{0}\right)^{2}+P^{2}-2 p^{0} Q-2 P Q x+m^{2}}\right],
\end{aligned}
$$

where $Q=|\mathbf{q}|, P=|\mathbf{p}|, x=\cos \theta$,

$$
\begin{aligned}
g_{2}\left(p^{2}, q^{2}, p \cdot q\right) & \\
= & g_{1}\left(p^{2},(p-q)^{2}, p \cdot(p-q)\right) \\
= & -2 m^{4}-2 m^{2} p^{2}+p^{4}+2 m^{2}(p \cdot q) \\
& -2 p^{2}(p \cdot q)+p^{2} q^{2},
\end{aligned}
$$

and we have performed the trivial angular integration over $\phi$. We now integrate with respect to $x$ to get

$$
\begin{aligned}
A(p)= & \int_{0}^{\infty} \frac{n(Q) \mathrm{d} Q}{16 \pi^{2} P}\left[8 P Q\left[-m^{2}-\left(p^{0}\right)^{2}+P^{2}\right]+m^{2}\left\{m^{2}+2\left[\left(p^{0}\right)^{2}-P^{2}\right]\right\}\right. \\
& \left.\times \ln \left(\frac{\left[m^{2}-\left(p^{0}-P+2 Q\right)\left(p^{0}+P\right)\right]\left[m^{2}-\left(p^{0}-P\right)\left(p^{0}+P-2 Q\right)\right]}{\left[m^{2}-\left(p^{0}-P-2 Q\right)\left(p^{0}+P\right)\right]\left[m^{2}-\left(p^{0}-P\right)\left(p^{0}+P+2 Q\right)\right]}\right)\right] .
\end{aligned}
$$

The result of this integral cannot be given in closed analytic form, in general. However in this paper we are mainly interested in its on-shell value $p^{0}=E_{\mathbf{p}}=\sqrt{m^{2}+P^{2}}$, and in this limit the integral can be computed exactly at any temperature since the logarithmic term in Eq. (D2) vanishes. In this case the value of the integral is given by

$$
A\left(E_{\mathbf{p}}, \mathbf{p}\right)=-\frac{m^{2}}{\pi^{2}} \int_{0}^{\infty} \mathrm{d} Q n(Q) Q=-\frac{1}{6} m^{2} T^{2},
$$

where we used that

$$
\int_{0}^{\infty} \mathrm{d} Q n(Q) Q=\frac{\pi^{2} T^{2}}{6} .
$$

We now proceed with the computation of $B(p)$, defined in Eq. (25b). Repeating similar steps as in the previous integral we get

$$
\begin{aligned}
B(p)= & \int_{0}^{\infty} \frac{n\left(E_{\mathbf{k}}\right) K^{2} \mathrm{~d} K}{2(2 \pi)^{2} E_{\mathbf{k}}} \\
& \times \int_{-1}^{1} \mathrm{~d} x\left[\mathrm{P} \frac{g_{1}\left(p^{2}, 0,-p^{0} E_{\mathbf{k}}+P K x\right)}{-\left(p^{0}\right)^{2}+P^{2}+2 p^{0} E_{\mathbf{k}}-2 P K x}\right. \\
& \left.+\mathrm{P} \frac{g_{1}\left(p^{2}, 0, p^{0} E_{\mathbf{k}}+P K x\right)}{-\left(p^{0}\right)^{2}+P^{2}-2 p^{0} E_{\mathbf{k}}-2 P K x}\right],
\end{aligned}
$$

where we recall that $E_{\mathbf{k}}=\sqrt{m^{2}+K^{2}}$. The integral with respect to $x$ can be analytically performed; the result is a very large and cumbersome expression, which we shall not reproduce here. The resulting expression cannot be integrated again in a closed analytic form. However, we may find par- ticular expressions valid at low and high temperatures. As in the case of previous integral, we will restrict to the on-shell results.

At low temperature, only those momenta $\mathbf{k}$ whose corresponding energies are at most of the order of the temperature, $E_{\mathbf{k}} \leq T$, contribute significatively to the integral because of the presence of the thermal factor $n\left(E_{\mathbf{k}}\right)$, which acts as a soft cutoff. Hence, low temperature also implies low energy and low momentum. Therefore, in the low-temperature approximation we may retain only the leading term in a $K$ expansion:

$$
B\left(E_{\mathbf{p}}, \mathbf{p}\right)=\frac{m^{2}+2 P^{2}}{\pi^{2}\left(3 m^{2}+4 P^{2}\right)} \int_{0}^{\infty} \mathrm{d} K n\left(E_{\mathbf{k}}\right)\left[K^{2}+O\left(K^{3}\right)\right] .
$$

Taking into account that for low temperatures

$$
n\left(E_{\mathbf{k}}\right) \approx e^{-m / T} e^{-K^{2} /(2 m T)}
$$

and that

$$
\int_{0}^{\infty} \mathrm{d} K e^{-K^{2} /(2 m T)} K^{2}=\sqrt{\frac{\pi}{2}}(m T)^{3 / 2}
$$

we find the following expression for $B(p)$ at low temperature:

$$
B\left(E_{\mathbf{p}}, \mathbf{p}\right) \approx \sqrt{\frac{m^{5} T^{3}}{2 \pi^{3}}}\left(\frac{m^{2}+2 P^{2}}{3 m^{2}+4 P^{2}}\right) e^{-m / T} .
$$

We have not made precise the exact meaning of the "lowtemperature" approximation employed above. In principle this approximation would require the temperature $T$ to be much smaller than any relevant quantity with dimensions of 
energy that could be formed by a combination of $m$ and $P$. However, a detailed analysis of the expressions shows that the condition $m \gg T$ is sufficient to guarantee the validity of the result.

Let us now proceed to the calculation of $B(p)$ in the high-temperature regime. Since $B(p)$ would be divergent if no thermal cutoff were present, at high temperatures the leading contribution to the integral is given by those momenta close to the temperature $T$. Thus as a first approximation we can retain only the leading term in a $1 / K$ expansion:

$$
\begin{aligned}
B\left(E_{\mathbf{p}}, \mathbf{p}\right)= & {\left[\frac{m^{2} \sqrt{m^{2}+P^{2}}}{4 \pi^{2} P} \ln \left(\frac{2 \sqrt{m^{2}+P^{2}}-P}{2 \sqrt{m^{2}+P^{2}}+P}\right)+\frac{3}{4 \pi^{2}} m^{2}\right] } \\
& \times \int_{0}^{\infty} \mathrm{d} K n\left(E_{\mathbf{k}}\right)\left[K+O\left(1 / K^{0}\right)\right] .
\end{aligned}
$$

Since the leading contribution to the integral is given in the ultrarelativistic regime, we can approximate the energy by the momentum in the Bose-Einstein function, $n\left(E_{\mathbf{k}}\right)$ $\approx n(K)$. With this approximation we find

$$
B\left(E_{\mathbf{p}}, \mathbf{p}\right)=\frac{m^{2} T^{2} E_{\mathbf{p}}}{24 P} \ln \left(\frac{2 E_{\mathbf{p}}-P}{2 E_{\mathbf{P}}+P}\right)+\frac{1}{8} m^{2} T^{2} .
$$

Analogously to the low-temperature case, the hightemperature approximation would $a$ priori require the temperature $T$ to be much higher than $m, P$, and any relevant energy scale formed by combination of these two. Again, one can show that the condition $T \gg m$ is sufficient to guarantee the validity of Eq. (D6).

We now move to the integral $C\left(p^{2}\right)$, defined in Eq. (25c). Its evaluation is straightforward:

$$
C\left(p^{2}\right)=g_{3}\left(p^{2}\right) \int_{0}^{\infty} \frac{K \mathrm{~d} K}{2 \pi^{2}} n(K)=\frac{T^{2}}{12}\left(10 m^{2}+4 p^{2}\right) .
$$

We only need its on-shell value $C\left(-m^{2}\right)=T^{2} m^{2} / 6$.

Let us now consider the integral $D(p)$, defined in Eq. (34). We start by introducing the variable $q=p-k$ :

$$
\begin{aligned}
D(p)= & \int \frac{\mathrm{d} q^{0} \mathrm{~d}^{3} \mathbf{q}}{(2 \pi)^{2}} F\left(p^{0}, q^{0}\right) g_{2}\left(-\left(p^{0}\right)^{2}+|\mathbf{p}|^{2},-\left(q^{0}\right)^{2}+\mathbf{q}^{2},\right. \\
& \left.-p^{0} q^{0}+\mathbf{p} \cdot \mathbf{q}\right) \delta\left(-\left(q^{0}\right)^{2}+\mathbf{q}^{2}\right) \delta\left(-\left(p^{0}-q^{0}\right)^{2}\right. \\
& \left.+(\mathbf{p}-\mathbf{q})^{2}+m^{2}\right),
\end{aligned}
$$

where $p=\left(p^{0}, \mathbf{p}\right)$ and $q=\left(q^{0}, \mathbf{q}\right)$. Next we expand the first delta function and integrate over $q^{0}$ :

$$
\begin{aligned}
D(p)= & \int \frac{\mathrm{d}^{3} \mathbf{q}}{(2 \pi)^{3}} \frac{2 \pi}{2|\mathbf{q}|} F\left(p^{0},|\mathbf{q}|\right) g_{2}\left(|\mathbf{p}|^{2}-\left(p^{0}\right)^{2}, 0, \mathbf{p} \cdot \mathbf{q}\right. \\
& \left.-p^{0}|\mathbf{q}|\right) \delta\left(-\left(p^{0}-|\mathbf{q}|\right)^{2}+(\mathbf{p}-\mathbf{q})^{2}+m^{2}\right) \\
& +\int \frac{\mathrm{d}^{3} \mathbf{q}}{(2 \pi)^{3}} \frac{2 \pi}{2|\mathbf{q}|} F\left(p^{0},-|\mathbf{q}|\right) g_{2}\left(|\mathbf{p}|^{2}-\left(p^{0}\right)^{2}, 0, \mathbf{p} \cdot \mathbf{q}\right. \\
& \left.+p^{0}|\mathbf{q}|\right) \delta\left(-\left(p^{0}+|\mathbf{q}|\right)^{2}+(\mathbf{p}-\mathbf{q})^{2}+m^{2}\right) .
\end{aligned}
$$

We now introduce spherical coordinates over $\mathbf{q}$ and expand the second delta function:

$$
\begin{aligned}
D(p)= & \frac{1}{8 \pi P} \int_{-1}^{1} \mathrm{~d} x \int_{0}^{\infty} \mathrm{d} Q F\left(p^{0}, Q\right) g_{2}\left(P^{2}-\left(p^{0}\right)^{2}, 0, Q P x\right. \\
& \left.-Q p^{0}\right) \delta\left(x-\left(p^{2}+2 p^{0} Q+m^{2}\right) /(2 P Q)\right) \\
& +\frac{1}{8 \pi P} \int_{-1}^{1} \mathrm{~d} x \int_{0}^{\infty} \mathrm{d} Q F\left(p^{0},-Q\right) \\
& \times g_{2}\left(P^{2}-\left(p^{0}\right)^{2}, 0, Q P x+Q p^{0}\right) \\
& \times \delta\left(x-\left(p^{2}-2 p^{0} Q+m^{2}\right) /(2 P Q)\right),
\end{aligned}
$$

where $Q=|\mathbf{q}|, P=|\mathbf{p}|$, and $x=\cos \theta$. We have already performed the trivial angular integration over $\phi$. Integrating with respect to $x$ with the aid of the delta function we get

$$
D(p)=\frac{g_{2}\left(p^{2}, 0,\left(p^{2}+m^{2}\right) / 2\right)}{8 \pi P}\left|\int_{Q_{1}}^{Q_{2}} \mathrm{~d} Q F\left(p^{0}, Q\right)\right|,
$$

with

$$
Q_{2}=\frac{\left(p^{0}\right)^{2}-P^{2}-m^{2}}{2\left(p^{0}-P\right)}, \quad Q_{1}=\frac{\left(p^{0}\right)^{2}-P^{2}-m^{2}}{2\left(p^{0}+P\right)}
$$

which can be finally arranged as

$$
D(p)=\frac{-m^{2}\left(m^{2}+2 p^{2}\right)}{8 \pi P}\left|\int_{Q_{1}}^{Q_{2}} \mathrm{~d} Q F\left(p^{0}, Q\right)\right| .
$$

Recall that $p^{2}=-\left(p^{0}\right)^{2}+P^{2}$.
[1] T. Jacobson, Phys. Rev. D 44, 1731 (1991).

[2] T. Jacobson, Phys. Rev. D 48, 728 (1993).

[3] T. Jacobson, Suppl. Prog. Theor. Phys. 136, 1 (1999).

[4] W.G. Unruh, Phys. Rev. D 51, 2827 (1995).

[5] R. Brout, S. Massar, R. Parentani, and P. Spindel, Phys. Rev. D 52, 4559 (1995).

[6] S. Corley and T. Jacobson, Phys. Rev. D 54, 1568 (1996).

[7] A.D. Helfer, Rep. Prog. Phys. 66, 943 (2003).
[8] J. Martin and R.H. Brandenberger, Phys. Rev. D 63, 123501 (2001).

[9] J.C. Niemeyer, Phys. Rev. D 63, 123502 (2001).

[10] J.C. Niemeyer and R. Parentani, Phys. Rev. D 64, 101301 (2001).

[11] J. Martin and R. Brandenberger, Phys. Rev. D 68, 063513 (2003).

[12] W.G. Unruh, Phys. Rev. Lett. 46, 1351 (1981). 
[13] M. Visser, Class. Quantum Grav. 15, 1767 (1998).

[14] L.J. Garay, J.R. Anglin, J.I. Cirac, and P. Zoller, Phys. Rev. Lett. 85, 4643 (2000).

[15] M. Visser, C. Barceló, and S. Liberati, Gen. Relativ. Gravit. 34, 1719 (2002).

[16] G. 't Hooft, Mod. Phys. Lett. A A 11, 4623 (1996).

[17] R. Parentani, Phys. Rev. D 63, 041503 (2001).

[18] R. Parentani, Int. J. Theor. Phys. 41, 2175 (2002).

[19] M. Takeda et al., Phys. Rev. Lett. 81, 1163 (1998).

[20] T. Kifune, Astrophys. J. Lett. 518, L21 (1999).

[21] G. Amelino-Camelia and T. Piran, Phys. Lett. B 497, 265 (2001).

[22] G. Amelino-Camelia and T. Piran, Phys. Rev. D 64, 036005 (2001).

[23] J. Alfaro, H.A. Morales-Tecotl, and L.F. Urrutia, Phys. Rev. Lett. 84, 2318 (2000).

[24] J. Alfaro, H.A. Morales-Tecotl, and L.F. Urrutia, Phys. Rev. D 65, 103509 (2002).

[25] V.A. Kostelecký and S. Samuel, Phys. Rev. D 39, 683 (1989).

[26] S.M. Carroll, J.A. Harvey, V.A. Kostelecký, C.D. Lane, and T. Okamoto, Phys. Rev. Lett. 87, 141601 (2001).

[27] V.A. Kostelecký, R. Lehnert, and M.J. Perry, Phys. Rev. D 68, 123511 (2003).

[28] J.D. Bjorken, Phys. Rev. D 67, 043508 (2003).

[29] S.M. Carroll, G.B. Field, and R. Jackiw, Phys. Rev. D 41, 1231 (1990).

[30] V.A. Kostelecký and M. Mewes, Phys. Rev. Lett. 87, 251304 (2001).

[31] T. Jacobson, S. Liberati, and D. Mattingly, Phys. Rev. D 66, 081302 (2002).

[32] T. Jacobson, S. Liberati, and D. Mattingly, Phys. Rev. D 67, 124011 (2003).

[33] T. Jacobson, S. Liberati, and D. Mattingly, Nature (London) 424, 1019 (2003).

[34] T. Jacobson, S. Liberati, D. Mattingly, and F.W. Stecker, Phys. Rev. Lett. 93, 021101 (2004).

[35] B. Hatfield, Quantum Field Theory of Point Particles and Strings (Addison-Wesley, Redwood City, CA, 1992).

[36] S. Weinberg, Foundations, The Quantum Theory of Fields, Vol. I (Cambridge University Press, Cambridge, England, 1995).

[37] J.F. Donoghue, B.R. Holstein, and R.W. Robinett, Ann. Phys. (N.Y.) 164, 233 (1985)

[38] H.A. Weldon, Phys. Rev. D 28, 2007 (1983).

[39] H.A. Weldon, Phys. Rev. D 26, 2789 (1982).

[40] J.F. Donoghue and B.R. Holstein, Phys. Rev. D 28, 340 (1983).

[41] H.A. Weldon, Phys. Rev. D 59, 065002 (1999).

[42] R. Tarrach, Phys. Lett. 133B, 259 (1983).

[43] G. Barton, Phys. Lett. B 237, 559 (1990).

[44] J.I. Latorre, P. Pascual, and R. Tarrach, Nucl. Phys. B437, 60 (1995).

[45] I.T. Drummond and S.J. Hathrell, Phys. Rev. D 22, 343 (1980).

[46] G.M. Shore, Nucl. Phys. B633, 271 (2002).

[47] G.M. Shore, gr-qc/0302116.

[48] G.M. Shore, Nucl. Phys. B646, 281 (2002).
[49] G.M. Shore, Contemp. Phys. 44, 503 (2003).

[50] S. Weinberg, Physica A 96, 327 (1979).

[51] J.F. Donoghue, Phys. Rev. Lett. 72, 2996 (1994).

[52] J.F. Donoghue, Phys. Rev. D 50, 3874 (1994).

[53] J. F. Donoghue, "Introduction to the effective field theory description of gravity," gr-qc/9512024.

[54] N.P. Landsman and C.G. van Weert, Phys. Rep. 145, 141 (1987).

[55] A. Das, Finite Temperature Field Theory (World Scientific, Singapore, 1997).

[56] M. le Bellac, Thermal Field Theory (Cambridge University Press, Cambridge, England, 1996).

[57] J.S. Schwinger, J. Math. Phys. 2, 407 (1961).

[58] L.V. Keldysh, Zh. Eksp. Teor. Fiz 47, 1515 (1965), [Sov. Phys. JETP 20, 1018 (1965)]

[59] K.-C. Chou, Z.-B. Su, B.-L. Hao, and L. Yu, Phys. Rep. 118, 1 (1985).

[60] E. Calzetta and B.L. Hu, Phys. Rev. D 49, 6636 (1994).

[61] B.L. Hu and A. Matacz, Phys. Rev. D 51, 1577 (1995).

[62] B.L. Hu and S. Sinha, Phys. Rev. D 51, 1587 (1995).

[63] A. Campos and E. Verdaguer, Phys. Rev. D 53, 1927 (1996).

[64] E. Calzetta, A. Campos, and E. Verdaguer, Phys. Rev. D 56, 2163 (1997).

[65] R. Martín and E. Verdaguer, Phys. Rev. D 60, 084008 (1999).

[66] R. Martín and E. Verdaguer, Phys. Lett. B 465, 113 (1999).

[67] R. Martín and E. Verdaguer, Phys. Rev. D 61, 124024 (2000).

[68] B.L. Hu and E. Verdaguer, Class. Quantum Grav. 20, R1 (2003).

[69] C. Barrabes, V. Frolov, and R. Parentani, Phys. Rev. D 62, 044020 (2000).

[70] E. Tomboulis, Phys. Lett. 70B, 361 (1977).

[71] A. Roura, Ph.D. thesis, Universitat de Barcelona, 2001.

[72] A. Roura and E. Verdaguer (in preparation).

[73] J. Borgman and L.H. Ford, gr-qc/0307043.

[74] C.P. Burgess, J. Cline, E. Filotas, J. Matias, and G.D. Moore, J. High Energy Phys. 03, 043 (2002).

[75] G. 't Hooft and M. Veltman, Ann. Inst. Henri Poincare, Sect. A 20, 69 (1974).

[76] M. Veltman, in Methods in Field Theory. Proceedings of the Les Houches Summer School 1975, edited by R. Balian and J. Zinn-Justin (North-Holland, Amsterdam, 1976), pp. 265-327.

[77] M. E. Peskin and D. V. Schroeder, An Introduction to Quantum Field Theory (Addison-Wesley, Reading, MA, 1998).

[78] G. Leibbrandt, Rev. Mod. Phys. 47, 849 (1975).

[79] P. Pascual and R. Tarrach, QCD: Renormalization for the Practitioner (Springer, Berlin, 1984).

[80] D. Arteaga, R. Parentani, and E. Verdaguer (in preparation).

[81] A. Rebhan, Phys. Rev. D 46, 4779 (1992).

[82] R. Lehnert, Phys. Rev. D 68, 085003 (2003).

[83] A. Campos and B. L. Hu, Phys. Rev. D 58, 125021 (1998).

[84] A. L. Fetter and J. D. Walecka, Quantum Theory of ManyParticle Systems (McGraw-Hill, New York, 1971).

[85] M.A. van Eijck, R. Kobes, and C.G. van Weert, Phys. Rev. D 50, 4097 (1994). 